\title{
Analytical results for the distribution of first hitting times of random walks on random regular graphs
}

\author{
Ido Tishby, Ofer Biham and Eytan Katzav \\ Racah Institute of Physics, The Hebrew University, Jerusalem 9190401, Israel. \\ E-mail: ido.tishby@mail.huji.ac.il, biham@phys.huji.ac.il, \\ eytan.katzav@mail.huji.ac.il
}

\begin{abstract}
.
We present analytical results for the distribution of first hitting times of random walks (RWs) on random regular graphs (RRGs) of degree $c \geq 3$ and a finite size $N$. Starting from a random initial node at time $t=1$, at each time step $t \geq 2$ an RW hops randomly into one of the $c$ neighbors of its previous node. In some of the time steps the RW may hop into a yet-unvisited node while in other time steps it may revisit a node that has already been visited before. The first time at which the RW enters a node that has already been visited before is called the first hitting time or the first intersection length. The first hitting event may take place either by backtracking (BT) to the previous node or by retracing (RET), namely stepping into a node which has been visited two or more time steps earlier. We calculate the tail distribution $P\left(T_{\mathrm{FH}}>t\right)$ of first hitting $(\mathrm{FH})$ times as well as its mean $\left\langle T_{\mathrm{FH}}\right\rangle$ and variance $\operatorname{Var}\left(T_{\mathrm{FH}}\right)$. We also calculate the probabilities $P_{\mathrm{BT}}$ and $P_{\mathrm{RET}}$ that the first hitting event will occur via the backtracking scenario or via the retracing scenario, respectively. We show that in dilute networks the dominant first hitting scenario is backtracking while in dense networks the dominant scenario is retracing and calculate the conditional distributions $P\left(T_{\mathrm{FH}}=t \mid \mathrm{BT}\right)$ and $P\left(T_{\mathrm{FH}}=t \mid \mathrm{RET}\right)$, for the two scenarios. The analytical results are in excellent agreement with the results obtained from computer simulations. Considering the first hitting event as a termination mechanism of the RW trajectories, these results provide useful insight into the general problem of survival analysis and the statistics of mortality rates when two or more termination scenarios coexist.
\end{abstract}

Keywords: Random network, random regular graph, random walk, backtracking, retracing, first hitting time, first intersection length. 


\section{Introduction}

Random walk (RW) models [1,2] are useful for the study of a large variety of stochastic processes such as diffusion [3, 4], polymer structure [5] 7], and random search [8, 9]. These models were studied extensively in different geometries, including continuous space [10], regular lattices [11, fractals [12] and random networks [13]. In the context of complex networks [14,15], random walks provide useful tools for the analysis of dynamical processes such as the spreading of rumours, opinions and infections [16, 17.

Consider an RW on a random network. Starting at time $t=1$ from a random initial node $x_{1}$, at each time step $t \geq 2$ it hops randomly to one of the neighbors of the previous node. The RW thus generates a trajectory of the form $x_{1} \rightarrow x_{2} \rightarrow \cdots \rightarrow x_{t} \rightarrow \ldots$, where $x_{t}$ is the node visited at time $t$. In some of the time steps the RW hops into nodes that have not been visited before, while in other time steps it hops into nodes that have already been visited at an earlier time. Since RWs on random networks may visit some of the nodes more than once, the number of distinct nodes visited up to time $t$ cannot exceed $t$ and is typically smaller than $t$. The mean number $\langle S\rangle_{t}$ of distinct nodes visited by an RW on a random network up to time $t$ was recently studied [18. It was found that in the infinite network limit it scales linearly with $t$, namely $\langle S\rangle_{t} \simeq r t$, where the coefficient $r<1$ depends on the network topology. These scaling properties resemble those obtained for RWs on high dimensional lattices and Cayley trees and imply that RWs on random networks revisit previously visited nodes less frequently than RWs on low dimensional lattices [19]. Therefore, RW models provide a highly effective framework for search and exploration processes on random networks.

The early stages of an RW trajectory can be characterized by the distribution of first hitting $(\mathrm{FH})$ times $P\left(T_{\mathrm{FH}}=t\right)$, where $T_{\mathrm{FH}}$ is the first time at which the RW steps into a node which has already been visited before. The first hitting time is also known as the first intersection length [20,21]. The first hitting event may occur either via the backtracking scenario, in which the RW hops back into the previous node, or by the retracing scenario, in which it hops into a node visited two or more time steps earlier. The first hitting event marks the transition from the first stage to the second stage in the life cycle of an RW. In the first stage the RW visits a new node at each time step, while in the second stage it combines moves at which the RW visits new nodes and moves at which it enters nodes that have already been visited before. The second stage in the life cycle of an RW on a finite network can be characterized by the distribution of first passage $(\mathrm{FP})$ times $P\left(T_{\mathrm{FP}}=t\right)$, where $T_{\mathrm{FP}}$ is the first time at which an $\mathrm{RW}$ starting from a random initial node $i$ visits a random target node $j$ [22,23]. Finally, the cover time, at which the RW completes visiting all the $N$ nodes in the network at least once marks the transition from the second stage to the third stage in the life cycle of an RW [24]. Beyond the cover time the RW continues to revisit nodes that have already been visited before.

Another type of random walk model is the non-backtracking random walk (NBW). At each time step the NBW steps into a random neighbor of the present node, except for 
the node visited in the previous time step. It is thus similar to the RW, except for the backtracking step which is eliminated. The paths of NBWs have been studied on regular lattices and random graphs [25]. It was shown that they explore the network more efficiently than RWs. It was also shown that they mix faster, namely require a shorter transient time to reach the stationary distribution of visiting frequencies throughout the network.

In a recent paper we presented analytical results for the distribution of first hitting times of RWs on Erdős-Rényi (ER) networks [26]. It was found that the tail distribution of first hitting times, $P\left(T_{\mathrm{FH}}>t\right)$, consists of a product of a geometric distribution due to the backtracking process and a Rayleigh distribution due to the retracing process. The mean and variance of the distribution of first hitting times were also calculated. In this analysis we utilized a special property of RWs on ER networks, in which the subnetwork that consists of the yet-unvisited nodes remains an ER network at all times. Its degree distribution remains a Poisson distribution, while its mean degree decreases linearly with the time $t$. This self-similarity enabled us to calculate the probability that at time $t$ the RW will step into a yet-unvisited node and the complementary probability that it will step into an already visited node. In the case of other configuration model networks, there is no closed form expression for the time evolution of the degree distribution of the sub-network of the yet-unvisited nodes. Therefore, the approach we used for the calculation of the distribution of first hitting times in ER networks cannot be generalized to other configuration model networks. However, it turns out that in the special case of random regular graphs there are other simplifying features that can be used to derive a closed form expression for $P\left(T_{\mathrm{FH}}>t\right)$.

In another recent paper we studied the distribution of first hitting times of NBWs on ER networks [27. Apart from the retracing scenario, NBWs on ER networks exhibit an additional mechanism of first hitting, referred to as the trapping scenario. The trapping takes place when the NBW enters a leaf node of degree $k=1$, which has no other neighbor except from the previous node. It was found that the tail distribution of first hitting times of NBWs on ER networks consists of a product of a geometric distribution due to the trapping process and a Rayleigh distribution due to the retracing process. The mean and variance of the distribution of first hitting times were also calculated.

In this paper we present analytical results for the distributions of first hitting times of random walks on random regular graphs (RRGs) of degree $c \geq 3$ and a finite size $N$. The first hitting event may take place either by backtracking to the previous node or by retracing, namely stepping into a node which has been visited two or more time steps earlier. Using the microstructure and statistical properties of RW paths at early times, we calculate the tail distribution of first hitting $(\mathrm{FH})$ times $P\left(T_{\mathrm{FH}}>t\right)$, which is given by a product of a geometric distribution due to the backtracking process and a Rayleigh distribution due to the retracing process. We also obtain closed form expressions for the mean first hitting time $\left\langle T_{\mathrm{FH}}\right\rangle$ and for the variance $\operatorname{Var}\left(T_{\mathrm{FH}}\right)$ of the distribution of first hitting times. The analytical results are found to be in excellent agreement with the results obtained from computer simulations. We obtain analytical results for the 
probabilities $P_{\mathrm{BT}}$ and $P_{\mathrm{RET}}$ that the first hitting event will occur via the backtracking or retracing scenarios, respectively. We show that in dilute networks the dominant first hitting scenario is backtracking while in dense networks the dominant scenario is retracing. We also obtain expressions for the conditional distributions of first hitting time, $P\left(T_{\mathrm{FH}}=t \mid \mathrm{BT}\right)$ and $P\left(T_{\mathrm{FH}}=t \mid \mathrm{RET}\right)$, namely conditioned on the first hitting event occurring via the backtracking or the retracing scenario, respectively.

The paper is organized as follows. In Sec. 2 we briefly describe the random regular graph. In Sec. 3 we present the random walk model. In Sec. 4 we calculate the distribution of first hitting times. In Sec. 5 we calculate the mean first hitting time. In Sec. 6 we calculate the variance of the distribution of first hitting times. In Sec. 7 we analyze the interplay between the backtracking and retracing scenarios. In Sec. 8 we compare the results presented in this paper for RWs on RRGs with previous results for RWs on ER networks. The results are discussed in Sec. 9 and summarized in Sec. 10.

\section{The random regular graph}

A random network (or graph) consists of a set of $N$ nodes that are connected by edges in a way that is determined by some random process. For example, in a configuration model network the degree of each node is drawn independently from a given degree distribution $P(k)$ and the connections are random and uncorrelated [30].

An important example of a configuration model network is the Erdős-Rényi (ER) network [31 33]. The ER network, denoted by $\operatorname{ER}(N, p)$, consists of $N$ nodes such that each pair of nodes is connected with probability $p$. The degree distribution of an ER network is a binomial distribution, $B(N, p)$. In the limit $N \rightarrow \infty$ and $p \rightarrow 0$, where the mean degree $c=(N-1) p$ is held fixed, it converges to a Poisson distribution. In the asymptotic limit $(N \rightarrow \infty)$, the ER network exhibits a phase transition at $c=1$ (a percolation transition), such that for $c<1$ the network consists only of small clusters and isolated nodes, while for $c>1$ there is a giant cluster which includes a macroscopic fraction of the network, in addition to the small clusters and isolated nodes [28, 29]. At a higher value of the connectivity, namely at $c=\ln N$, there is a second transition, above which the entire network is included in the giant cluster and there are no isolated components.

The RRG is a special case of a configuration model network, in which the degree distribution is a degenerate distribution of the form $P(k)=\delta_{k, c}$, namely all the nodes are of the same degree $c$. Here we focus on the case of $3 \leq c \leq N-1$, in which for a sufficiently large value of $N$ the RRG consists of a single connected component. In the infinite network limit the RRG exhibits a tree structure with no cycles. Thus, in this limit it coincides with a Bethe lattice whose coordination number is equal to $c$. In contrast, RRGs of a finite size exhibit a local tree-like structure, while at larger scales there is a broad spectrum of cycle lengths [34]. In that sense RRGs differ from Cayley trees, which maintain their tree structure by reducing the most peripheral nodes to leaf nodes of degree 1 . 
A convenient way to construct an RRG of size $N$ and degree $c$ is to prepare the $N$ nodes such that each node is connected to $c$ half edges or stubs [15]. At each step of the construction, one connects a random pair of stubs that belong to two different nodes $i$ and $j$ that are not already connected, forming an edge between them. This procedure is repeated until all the stubs are exhausted. The process may get stuck before completion in case that all the remaining stubs belong to the same node or to pairs of nodes that are already connected. In such case one needs to perform some random reconnections in order to complete the construction.

RRGs provide a useful benchmark for the study of dynamical processes on regular lattices. This is due to the fact that for any regular lattice structure in any space dimension, one can construct an RRG whose degree $c$ is equal to the coordination number $z$ of the regular lattice. For example, a simple cubic lattice in a $d$ dimensional space corresponds to an RRG with degree $c=2 d$. Some special cases in two dimensions include the honeycomb lattice that corresponds to an RRG with $c=3$ and the triangular lattice that corresponds to an RRG with $c=6$. However, all the other structural properties of RRGs are completely different from those of the corresponding regular lattice. Therefore, comparing the behavior of dynamical processes taking place on regular lattices and on the corresponding RRGs, one can distinguish between the properties that depend on the coordination number and those that depend on other structural properties such as the lengths of short cycles. A famous example is the BethePeierls approximation of the Ising model [35], which is actually an exact result on an RRG with the same coordination number as a $d$-dimensional lattice. In 2 dimensions the critical exponents are wrongly identified as the mean-field ones, but the actual predictions for the critical temperature and the heat capacity are highly insightful. In dimensions higher than 4 , even the critical exponents are correct.

\section{The random walk model}

Consider an RW on an RRG of degree $c \geq 3$ and size $N$. At each time step the RW hops from its current node to one of its neighbors, such that the probability of hopping to each neighbor is $1 / c$. For sufficiently large $N$ the RRG consists of a single connected component, thus an RW starting from any initial node can reach any other node in the network. In the long time limit $t \gg N$ the RW visits all the nodes with the same frequency, namely on average each node is visited once every $N$ steps. However, over shorter periods of time there may be large fluctuations such that some nodes may be visited several times in a given time interval while other nodes are not visited at all.

In some of the time steps an RW may visit nodes that have not been visited before while in other time steps it may revisit nodes that have already been visited before. For example, at each time step $t \geq 3$ the RW may backtrack into the previous node with probability of $1 / c$. In the infinite network limit the RRG exhibits a tree structure. Therefore, in this limit the backtracking mechanism is the only way in which an RW may hop from a newly visited node to a node that has already been visited before. 
$(a)$

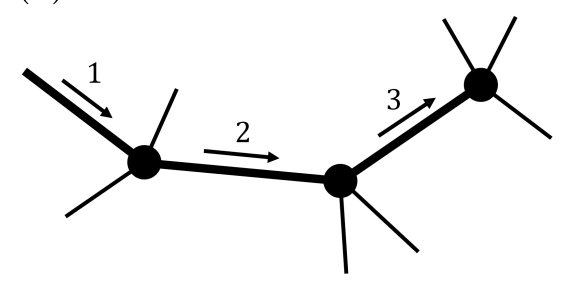

(b)
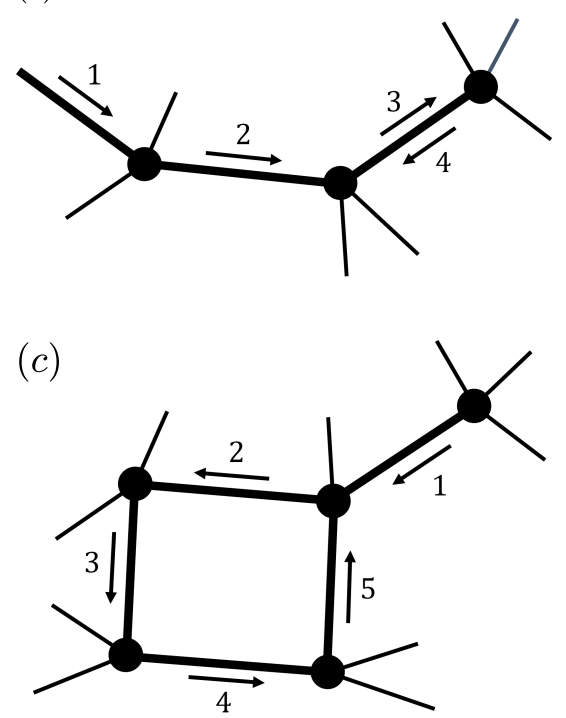

Figure 1. Schematic illustrations of possible events taking place along the path of an RW on an RRG: (a) a path segment in which at each time step the RW enters a node that has not been visited before; (b) a path segment that includes a backtracking step into the previous node (step no. 4); (c) a path that includes a retracing step (step no. 5) in which the RW hops into a node that was visited a few time steps earlier. Retracing steps are not possible in the infinite network limit and take place only in finite networks, which include cycles. Note that in this illustration the RRG is of degree $c=4$.

However, in finite networks the RW may utilize the cycles to retrace its path and hop into nodes that have already been visited two or more time steps earlier. Once the RW stepped into a node that has been visited before, it may continue to hop back and forth along the path of its previously visited nodes, until it eventually leaves the path and enters a newly visited node.

In Fig. 11 we present a schematic illustration of the backtracking and the retracing events which may take place along the path of an RW on an RRG. While backtracking is independent on the network size, retracing takes place only in finite networks and is not possible in the infinite network limit, in which the RRG exhibits a tree structure. 


\section{The distribution of first hitting times}

Consider an RW on an RRG of a finite size $N$ and degree $c \geq 3$. Starting from a random node at time $t=1$, the RW hops randomly between nearest neighbor nodes. At early times $t \ll N$ all the nodes it enters are likely to be visited for the first time. The first time at which the RW enters a node that has already been visited before is called the first hitting time. The first hitting process may take place either by backtracking (BT) or by retracing (RET). In the backtracking scenario the RW moves back into the previous node, while in the retracing scenario it hops into a node that has already been visited two or more time steps earlier. Below we calculate the distribution of first hitting times. In case that the RW has not returned to any previously visited node up to time $t-1$, the first hitting time satisfies the condition $T_{\mathrm{FH}}>t-1$. Given that $T_{\mathrm{FH}}>t-1$, the probability that the first hitting event will not take place in the next time step is expressed by the conditional probability $P\left(T_{\mathrm{FH}}>t \mid T_{\mathrm{FH}}>t-1\right)$. This conditional probability can be expressed as a product of the form

$$
P\left(T_{\mathrm{FH}}>t \mid T_{\mathrm{FH}}>t-1\right)=P_{t}(\neg \mathrm{RET} \mid \neg \mathrm{BT}) P_{t}(\neg \mathrm{BT}),
$$

where $P_{t}(\neg \mathrm{BT})$ is the probability that the RW will not backtrack to the previous node at time $t$. Given that the RW has not backtracked at time $t$, the conditional probability $P_{t}(\neg \mathrm{RET} \mid \neg \mathrm{BT})$ is the probability that it will also not retrace its path at time $t$, namely, that it will not hop into a node that has already been visited two or more time steps earlier.

Since all the nodes in the RRG are of degree $c$, at each time step (apart from $t=1$ and 2) the probability of backtracking into the previous node is $1 / c$. Therefore, the probability that a backtracking step will not occur at any given time $t \geq 3$ is

$$
P_{t}(\neg \mathrm{BT})=1-\frac{1}{c}
$$

Provided that the RW has not backtracked at time $t$, we will now evaluate the probability $P_{t}(\neg \mathrm{RET} \mid \neg \mathrm{BT})$ that it will also not retrace its path. Apart from the current node at time $t-1$ and the previous node (visited at time $t-2$ ), there are $N-2$ other nodes in the network, of which $t-3$ have already been visited by the RW.

Since the network is undirected, an edge connecting a pair of nodes $i$ and $j$ can be considered as a combination of two links, one from $i$ to $j$ and the other from $j$ to $i$. Thus, a node of degree $c$ is connected to $c$ incoming links and $c$ outgoing links. The number of yet-unvisited nodes at time $t-1$ is $N-t+1$. Each one of these nodes is of degree $c$.

Since at each time step the RW enters a node via one edge and leaves it via another edge, up to time $t-1$ it exhausts $t-2$ edges along the path. Thus, each one of the nodes visited in the first $t-3$ time steps exhibits only $c-2$ links that can be used by the RW to enter the node via the retracing mechanism at time $t$ (apart from the initial node that can be reached via $c-1$ edges). As a result, the number of incoming links 
that may lead the RW to an already visited node via the retracing mechanism at time $t$ is

$$
L_{\mathrm{v}}=(c-2)(t-3)+1 .
$$

The number of incoming links that may lead the RW to a yet-unvisited node at time $t$ is

$$
L_{\mathrm{u}}=(N-t+1) c .
$$

Summing up $L_{\mathrm{v}}$ and $L_{\mathrm{u}}$ we obtain the total number of incoming links that may lead the RW at time $t$ to either a visited node (by retracing) or to a yet-unvisited node. It is given by

$$
L_{\mathrm{T}}=(N-2) c-2(t-3)+1 .
$$

Given that the possibility of backtracking into the previous node was eliminated, the RW selects randomly one of the $c-1$ other neighbors of the current node. The probability that any one of these neighbors has already been visited before is thus given by $L_{\mathrm{v}} / L_{\mathrm{T}}$. Therefore, the probability of retracing at time $t$ under the condition of no-backtracking is given by

$$
P_{t}(\mathrm{RET} \mid \neg \mathrm{BT})=\frac{L_{\mathrm{v}}}{L_{\mathrm{T}}}=\frac{(c-2)(t-3)+1}{(N-2) c-2(t-3)+1} .
$$

In Fig. 2 we present analytical results, obtained from Eq. (6), for the probability $P_{t}(\mathrm{RET} \mid \neg \mathrm{BT})$ that an RW on an RRG will retrace its path for the first time at time $t$ under the condition that it has not backtracked its path at time $t$ (or earlier). The RRGs used in Fig. 2 are of size $N=1000$, where the nodes are of degree $c=3$ (solid line), 5 (dashed line) and 10 (dotted line). The analytical results are in excellent agreement with the results obtained from computer simulations (circles). These results are qualitatively different from the corresponding results for RWs on ER networks, which are given by [26]

$$
P_{t}^{\mathrm{ER}}(\mathrm{RET} \mid \neg \mathrm{BT})=\frac{c(t-3)}{(c+1)(N-1)},
$$

where $c$ is the mean degree.

Using Eq. (6), the probability that the RW will not retrace its path at time $t \geq 3$ is given by

$$
P_{t}(\neg \mathrm{RET} \mid \neg \mathrm{BT})=1-\frac{(c-2)(t-3)+1}{(N-2) c-2(t-3)+1} .
$$

Inserting the probabilities $P_{t}(\neg \mathrm{BT})$ and $P_{t}(\neg \mathrm{RET} \mid \neg \mathrm{BT})$ from Eqs. (2) and (8)), respectively, into Eq. (1), we obtain

$$
P\left(T_{\mathrm{FH}}>t \mid T_{\mathrm{FH}}>t-1\right)=\left(1-\frac{1}{c}\right)\left[1-\frac{(c-2)(t-3)+1}{(N-2) c-2(t-3)+1}\right] .
$$


First hitting times of random, walks on random reaular aranhs

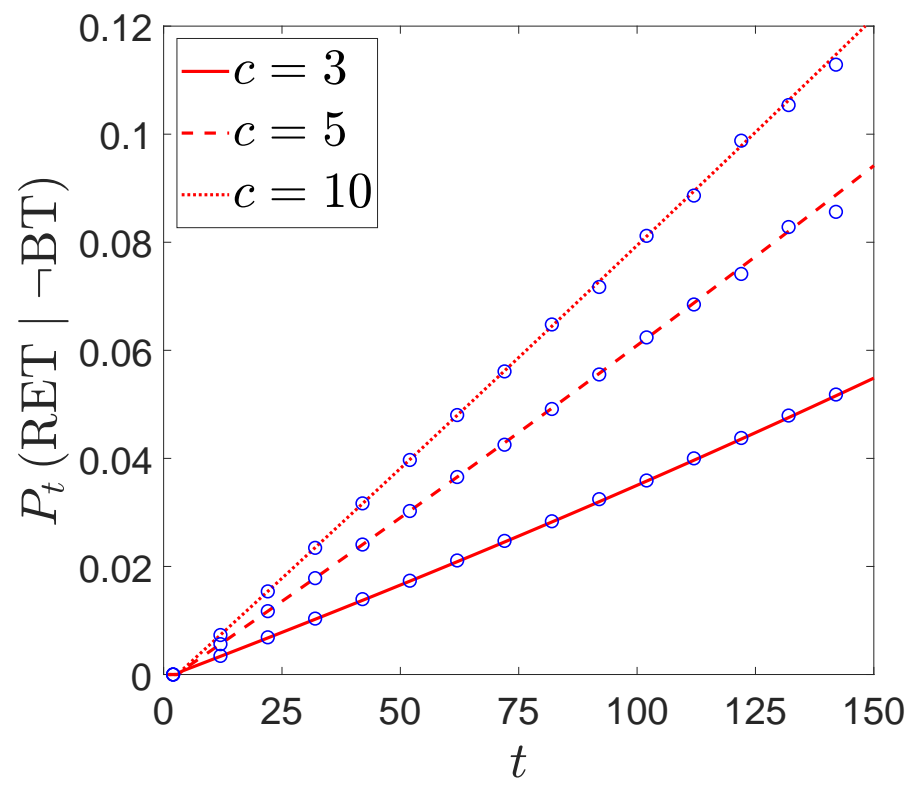

Figure 2. Analytical results for the probability $P_{t}(\mathrm{RET} \mid \neg \mathrm{BT})$ that an RW will retrace its path at time $t$ under the condition that it has not backtracked its path at time $t$, on an RRG of size $N=1000$ and $c=3$ (solid line), 5 (dashed line) and 10 (dotted line). The analytical results, obtained from Eq. (6), are in excellent agreement with the results obtained from computer simulations (circles).

Focusing on large networks, where $N \gg 1$, and assuming that $t \ll N c$, the probability $P_{t}(\neg \mathrm{RET} \mid \neg \mathrm{BT})$ can be written as a series expansion in powers of $(t-3) /\left(N_{c}\right)$. It is given by

$$
P_{t}(\neg \mathrm{RET} \mid \neg \mathrm{BT})=1-\frac{(c-2)(t-3)}{N c}-\frac{2(c-2)(t-3)^{2}}{(N c)^{2}}+\mathcal{O}\left[\left(\frac{t-3}{N c}\right)^{3}\right] .
$$

Using the expansion $\ln (1-x)=-x-x^{2} / 2+\mathcal{O}\left(x^{3}\right)$ for $x \ll 1$, we obtain

$$
\begin{aligned}
\ln \left[P_{t}(\neg \mathrm{RET} \mid \neg \mathrm{BT})\right] & \simeq-\frac{(c-2)(t-3)}{N c}-\frac{2(c-2)(t-3)^{2}}{(N c)^{2}} \\
& -\frac{1}{2}\left[\frac{(c-2)(t-3)}{N c}+\frac{2(c-2)(t-3)^{2}}{(N c)^{2}}\right]^{2} .
\end{aligned}
$$

Rearranging terms up to second order in $(t-3) /(N c)$, we obtain

$$
P_{t}(\neg \mathrm{RET} \mid \neg \mathrm{BT}) \simeq \exp \left[-\frac{(c-2)(t-3)}{N c}-\frac{(c-2)(c+2)(t-3)^{2}}{2(N c)^{2}}\right] .
$$

As a result, Eq. (9) is replaced by

$$
P\left(T_{\mathrm{FH}}>t \mid T_{\mathrm{FH}}>t-1\right) \simeq\left(1-\frac{1}{c}\right) \exp \left[-\frac{(c-2)(t-3)}{N c}-\frac{(c-2)(c+2)(t-3)^{2}}{2(N c)^{2}}\right],
$$


where $t \geq 3$.

The probability $P\left(T_{\mathrm{FH}}>t\right)$ that the first hitting event will not take place during the first $t$ time steps is given by the product

$$
P\left(T_{\mathrm{FH}}>t\right)=\prod_{t^{\prime}=3}^{t} P\left(T_{\mathrm{FH}}>t^{\prime} \mid T_{\mathrm{FH}}>t^{\prime}-1\right) .
$$

Inserting $P\left(T_{\mathrm{FH}}>t^{\prime} \mid T_{\mathrm{FH}}>t^{\prime}-1\right)$ from Eq. (13) into Eq. (14), we obtain the tail distribution of first hitting times

$$
P\left(T_{\mathrm{FH}}>t\right)=P_{\mathrm{BT}}\left(T_{\mathrm{FH}}>t\right) P_{\mathrm{RET}}\left(T_{\mathrm{FH}}>t\right)
$$

where

$$
P_{\mathrm{BT}}\left(T_{\mathrm{FH}}>t\right)= \begin{cases}1 & t=1,2 \\ \left(1-\frac{1}{c}\right)^{t-2} & t \geq 3\end{cases}
$$

and

$$
P_{\mathrm{RET}}\left(T_{\mathrm{FH}}>t\right) \simeq \begin{cases}1 & 1 \leq t \leq 3 \\ \prod_{t^{\prime}=3}^{t} \exp \left[-\frac{(c-2)\left(t^{\prime}-3\right)}{N c}-\frac{(c-2)(c+2)\left(t^{\prime}-3\right)^{2}}{2(N c)^{2}}\right] & t \geq 4\end{cases}
$$

Note that the contribution of the backtracking process, given by Eq. (16), depends only on the mean degree $c$, while the contribution of the retracing process, given by Eq. (17) depends on the mean degree $c$ and on the network size $N$. In the infinite network limit the retracing scenario becomes irrelevant and Eq. (15) is simplified to

$$
P\left(T_{\mathrm{FH}}>t \mid N \rightarrow \infty\right)= \begin{cases}1 & t=1,2 \\ \left(1-\frac{1}{c}\right)^{t-2} & t \geq 3 .\end{cases}
$$

Taking the logarithm of $P_{\mathrm{RET}}\left(T_{\mathrm{FH}}>t\right)$, as expressed in Eq. (17) for $t \geq 4$, we obtain

$$
\ln \left[P_{\mathrm{RET}}\left(T_{\mathrm{FH}}>t\right)\right] \simeq-\sum_{t^{\prime}=3}^{t} \frac{(c-2)\left(t^{\prime}-3\right)}{N c}-\sum_{t^{\prime}=3}^{t} \frac{(c-2)(c+2)\left(t^{\prime}-3\right)^{2}}{2(N c)^{2}}
$$

Carrying out the summations in Eq. (19), we obtain

$$
\ln \left[P_{\mathrm{RET}}\left(T_{\mathrm{FH}}>t\right)\right] \simeq-\frac{(c-2)(t-2)(t-3)}{2 N c}-\frac{(c-2)(c+2)(t-2)(t-3)(t-5 / 2)}{6(N c)^{2}}
$$

Inserting $P_{\mathrm{BT}}\left(T_{\mathrm{FH}}>t\right)$ from Eq. (16) and $P_{\mathrm{RET}}\left(T_{\mathrm{FH}}>t\right)$ from Eq. (20) into Eq. (15), we obtain 
First hitting times of random walks on random regular graphs

$$
P\left(T_{\mathrm{FH}}>t\right) \simeq \begin{cases}1 & t=1,2 \\ \exp \left[-\frac{(t-2)(t-3)}{2 \alpha^{2}}-\frac{(t-2)(t-3)(t-5 / 2)}{6\left(\frac{c-2}{c+2}\right) \alpha^{4}}-\beta(t-2)\right] & t \geq 3\end{cases}
$$

where the parameters $\alpha$ and $\beta$ are given by

$$
\alpha=\sqrt{\left(\frac{c}{c-2}\right) N}
$$

and

$$
\beta=\ln \left(\frac{c}{c-1}\right) .
$$

Using a Taylor expansion of the second term in the exponent in Eq. (21), we obtain

$$
P\left(T_{\mathrm{FH}}>t\right) \simeq \begin{cases}1 & t=1,2 \\ {\left[1-\frac{(t-2)(t-3)(t-5 / 2)}{6\left(\frac{c-2}{c+2}\right) \alpha^{4}}\right] \exp \left[-\frac{(t-2)(t-3)}{2 \alpha^{2}}-\beta(t-2)\right]} & t \geq 3\end{cases}
$$

where the first term in the second line is the leading term and the second term is a correction term, which is small for sufficiently small values of $t$.

It turns out that for large networks the second term in the second line of Eq. (24) is extremely small and its effect on the tail distribution $P\left(T_{\mathrm{FH}}>t\right)$ can be neglected in most cases. The only case in which it was found to make a small but noticeable difference is in the calculation of the second moment $\left\langle T_{\mathrm{FH}}^{2}\right\rangle$. For the sake of consistency, we use Eq. (24) in the calculations of both the mean first hitting time $\left\langle T_{\mathrm{FH}}\right\rangle$ and the second moment $\left\langle T_{\mathrm{FH}}^{2}\right\rangle$. In all the other calculations presented in this paper we use a simplified form of the tail distribution, which is given by

$$
P\left(T_{\mathrm{FH}}>t\right) \simeq \begin{cases}1 & t=1,2 \\ \exp \left[-\frac{(t-2)(t-3)}{2 \alpha^{2}}-\beta(t-2)\right] & t \geq 3\end{cases}
$$

The tail distribution $P\left(T_{\mathrm{FH}}>t\right)$, given by Eq. (25), is thus a product of a geometric distribution, associated with the backtracking process and a Rayleigh distribution, associated with the retracing process [36].

In Fig. 3 we present (on a semi-logarithmic scale) analytical results for the tail distributions $P\left(T_{\mathrm{FH}}>t\right)$ of first hitting times (solid lines) of RWs on RRGs of size $N=1000$ in which the nodes are of degree $c=3$ (left), 5 (middle) and 10 (right). The analytical results, obtained from Eq. (25) are in excellent agreement with the results obtained from computer simulations (circles). It is found that as the degree $c$ is increased the first hitting event tends to occur at a later time. This can be attributed to the fact that the probability of backtracking decreases as $c$ is increased. 

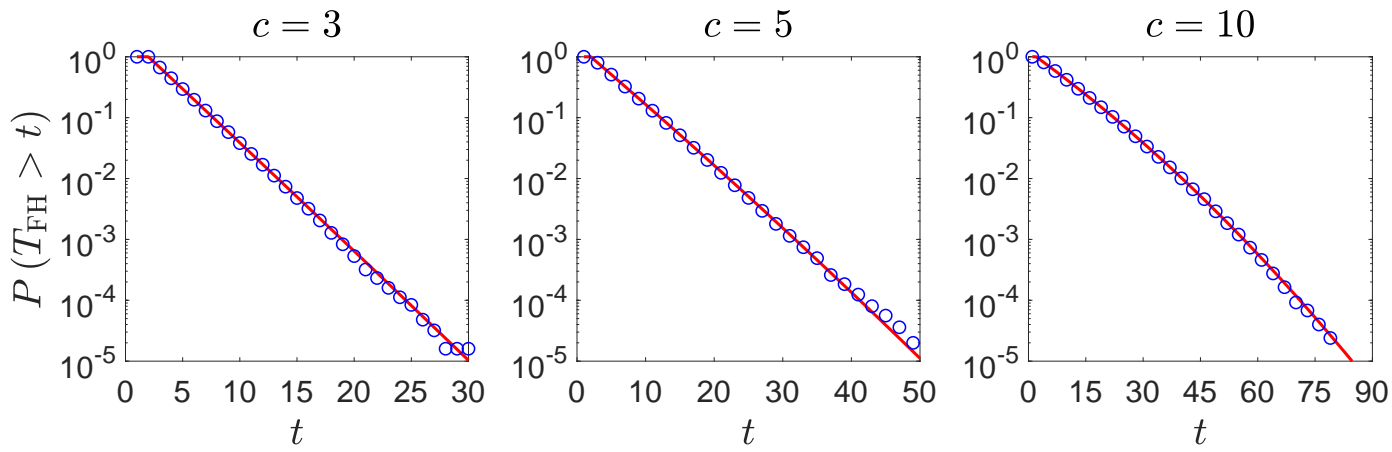

Figure 3. Analytical results for the tail distributions $P\left(T_{\mathrm{FH}}>t\right)$ of first hitting times (solid lines) of RWs on RRGs of size $N=1000$, in which the nodes are of degree $c=3$ (left), 5 (middle) and 10 (right). The analytical results, obtained from Eq. (25) are in excellent agreement with the results obtained from computer simulations (circles).

The excellent agreement observed in Fig. 3 between the analytical results and the results obtained from computer simulations indicates that Eq. (25) is valid for a broad range of parameters. However, Eq. (25) combines the backtracking and retracing mechanisms. Thus, for parameters in which the backtracking mechanism is dominant, Fig. 3 is not sufficient to establish the validity of the first term in Eq. (25), associated with the retracing scenario. This is due to the fact that in this regime the retracing process is effectively screened by the backtracking process.

In order to establish the validity of the retracing term in Eq. (25) we consider the distribution of first hitting times of NBWs on RRGs. The suppression of the backtracking process in NBWs leaves the retracing scenario as the only possible scenario of first hitting in these systems. This is unlike the case of NBWs on ER networks, in which the trapping scenario emerges once backtracking is suppressed. This contrast is due to the fact that RRGs do not include leaf nodes of degree $k=1$, in which an NBW may become trapped. The suppression of the backtracking mechanism implies that the distribution of first hitting times of NBWs on RRGs is expressed by the right hand side of Eq. (25), where $\alpha$ is given by Eq. (22) and $\beta=0$, namely

$$
P_{\mathrm{NBW}}\left(T_{\mathrm{FH}}>t\right) \simeq \begin{cases}1 & t=1,2 \\ \exp \left[-\frac{(t-2)(t-3)}{2 \alpha^{2}}\right] & t \geq 3 .\end{cases}
$$

In Fig. 4 we present (on a semi-logarithmic scale) analytical results for the tail distributions $P_{\mathrm{NBW}}\left(T_{\mathrm{FH}}>t\right.$ ) of first hitting times (solid lines) of NBWs on RRGs of size $N=1000$ in which the nodes are of degree $c=3$ (left), 5 (middle) and 10 (right). The analytical results, obtained from Eq. (26) are in excellent agreement with the results obtained from computer simulations (circles). 

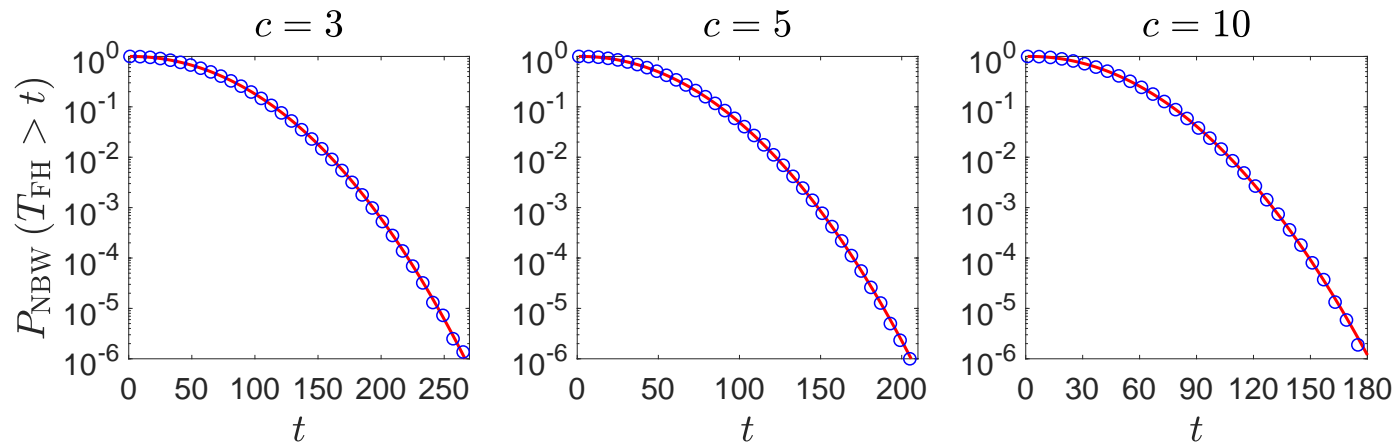

Figure 4. Analytical results for the tail distributions $P_{\mathrm{NBW}}\left(T_{\mathrm{FH}}>t\right)$ of first hitting times (solid lines) of NBWs on RRGs of size $N=1000$, in which the nodes are of degree $c=3$ (left), 5 (middle) and 10 (right). The analytical results, obtained from Eq. (26) are in excellent agreement with the results obtained from computer simulations (circles).

\section{The mean first hitting time}

The moments $\left\langle T_{\mathrm{FH}}^{r}\right\rangle, r=1,2, \ldots$, of the distribution of first hitting times of RWs on RRGs can be obtained from the tail-sum formula [37]

$$
\left\langle T_{\mathrm{FH}}^{r}\right\rangle=\sum_{t=0}^{N}\left[(t+1)^{r}-t^{r}\right] P\left(T_{\mathrm{FH}}>t\right) .
$$

In particular, the mean first hitting time $\left\langle T_{\mathrm{FH}}\right\rangle$ can be obtained by inserting $r=1$ in Eq. (27), which yields

$$
\left\langle T_{\mathrm{FH}}\right\rangle=\sum_{t=0}^{N} P\left(T_{\mathrm{FH}}>t\right) .
$$

Inserting $P\left(T_{\mathrm{FH}}>t\right)$ from Eq. (24) into Eq. (28), shifting the summation index from $t$ to $t-1$, and and replacing the sum in Eq. (28) by a sum of two integrals, using the formulation of a middle Riemann sum, we obtain

$$
\left\langle T_{\mathrm{FH}}\right\rangle \simeq I_{1}+I_{2},
$$

where

$$
I_{1}=3+e^{-\beta}+\int_{3 / 2}^{N-3 / 2} \exp \left[-\frac{t(t-1)}{2 \alpha^{2}}-\beta t\right] d t
$$

and

$$
I_{2}=-\int_{3 / 2}^{N-3 / 2} \frac{t(t-1)(t-1 / 2)}{6\left(\frac{c-2}{c+2}\right) \alpha^{4}} \exp \left[-\frac{t(t-1)}{2 \alpha^{2}}-\beta t\right] d t
$$


Carrying out the integration in Eq. (301), we obtain

$$
I_{1} \simeq 3+e^{-\beta}+\sqrt{\frac{\pi}{2}} \alpha \exp \left[\frac{\left(2 \alpha^{2} \beta-1\right)^{2}}{8 \alpha^{2}}\right]\left\{\operatorname{erf}\left[\frac{\alpha(\beta+1-2 / c)}{\sqrt{2}}\right]-\operatorname{erf}\left(\frac{\alpha^{2} \beta+1}{\sqrt{2} \alpha}\right)\right\}
$$

where $\operatorname{erf}(x)$ is the error function, also called Gauss error function [38]. The error function $\operatorname{erf}(x)$ is a monotonically increasing function, defined for $-\infty<x<\infty$. It is an odd function, namely $\operatorname{erf}(-x)=-\operatorname{erf}(x)$, and exhibits a sigmoidal shape. For $|x| \ll 1$ it can be approximated by $\operatorname{erf}(x) \simeq 2 x / \sqrt{\pi}$ while for $|x|>1$ it quickly converges to $\operatorname{erf}(x) \rightarrow \operatorname{sign}(x)$. The parameter $\beta$ obtains its largest value at $c=3$, where $\beta=\ln (3 / 2)$, and decreases monotonically as $c$ is increased. Thus, for any value of $c$ it satisfies $\beta<1$. In the limit of $c \gg 1$, it can be approximated by

$$
\beta=\frac{1}{c}+\frac{1}{2 c^{2}}+\mathcal{O}\left(\frac{1}{c^{3}}\right) .
$$

Since the degree $c$ satisfies $c<N-1$, the parameter $\beta$ is bounded from below by $\beta>1 /(N-1)$. In the limit of complete graph it approaches this lower bound, namely $\beta \rightarrow 1 /(N-1)$. The product $\alpha \beta$ thus takes values in the range $1 / \sqrt{N-2}<\alpha \beta \leq$ $\ln (3 / 2) \sqrt{N-2}$.

Eq. (32) can be written in the form

$$
\begin{aligned}
I_{1} \simeq 3+e^{-\beta}+ & \sqrt{\frac{\pi}{2}} \alpha \exp \left(\frac{-\beta}{2}\right) \exp \left(\frac{1}{8 \alpha^{2}}\right) \exp \left[\left(\frac{\alpha \beta}{\sqrt{2}}\right)^{2}\right] \times \\
& {\left[\operatorname{erf}\left(\frac{\alpha(1-2 / c)}{\sqrt{2}}+\frac{\alpha \beta}{\sqrt{2}}\right)-\operatorname{erf}\left(\frac{\alpha \beta}{\sqrt{2}}+\frac{1}{\sqrt{2} \alpha}\right)\right] . }
\end{aligned}
$$

In the large network limit, where $N \gg 1$, the term $\exp \left[1 /\left(8 \alpha^{2}\right)\right]$ satisfies $\exp \left[1 /\left(8 \alpha^{2}\right)\right] \simeq$ $1+\mathcal{O}(1 / N)$ and thus it can be neglected. Similarly, in the large network limit the argument of the first erf function in Eq. (32) is always very large. Therefore, one can safely set the first erf function to be equal to 1 . The second erf function can be approximated by

$$
\operatorname{erf}\left(\frac{\alpha \beta}{\sqrt{2}}+\frac{1}{\sqrt{2} \alpha}\right) \simeq \operatorname{erf}\left(\frac{\alpha \beta}{\sqrt{2}}\right)+\frac{\sqrt{2}}{\sqrt{\pi} \alpha} \exp \left[-\left(\frac{\alpha \beta}{\sqrt{2}}\right)^{2}\right]
$$

Making these approximations, we obtain

$$
I_{1} \simeq 3+e^{-\beta}-e^{-\beta / 2}+\sqrt{\frac{\pi}{2}} \alpha e^{\frac{-\beta}{2}} \exp \left[\left(\frac{\alpha \beta}{\sqrt{2}}\right)^{2}\right]\left[1-\operatorname{erf}\left(\frac{\alpha \beta}{\sqrt{2}}\right)\right] .
$$

Carrying out the integration in Eq. (31) and taking the large network limit, we obtain 


$$
\begin{aligned}
I_{2} & \simeq \frac{1}{6} \sqrt{\frac{\pi}{2}}\left(\frac{c+2}{c-2}\right) \alpha \beta\left(\alpha^{2} \beta^{2}+3\right) e^{\frac{\left(1-2 \alpha^{2} \beta\right)^{2}}{8 \alpha^{2}}}\left[1-\operatorname{erf}\left(\frac{\alpha^{2} \beta+1}{\sqrt{2} \alpha}\right)\right] \\
& -\frac{1}{6}\left(\frac{c+2}{c-2}\right)\left(\alpha^{2} \beta^{2}-\beta+2\right) e^{-\frac{3}{8 \alpha^{2}}-\frac{3 \beta}{2}} .
\end{aligned}
$$

In the dilute-network limit, where $3 \leq c \ll 1.63 \sqrt{N}$, the argument of the error function in Eq. (36) satisfies the condition $\alpha \beta / \sqrt{2} \gg 1$. In this limit one can use the approximation 38

$$
1-\operatorname{erf}(x) \simeq \frac{e^{-x^{2}}}{x \sqrt{\pi}},
$$

which is the first term in the asymptotic expansion of the complementary error function, and obtain

$$
\left\langle T_{\mathrm{FH}}\right\rangle \simeq c+2+\mathcal{O}\left(\frac{1}{c}\right) .
$$

This result reflects the fact that in the dilute network limit the first hitting time is dominated by the backtracking mechanism.

In the dense-network limit, where $1.63 \sqrt{N} \ll c \leq N$ the argument of the error function in Eq. (36) satisfies $\alpha \beta / \sqrt{2} \ll 1$. In this case, one can use the approximation $\operatorname{erf}(x) \simeq x$, which is the leading term in the Taylor expansion of $\operatorname{erf}(x)$ around $x=0$. Using this approximation, we obtain

$$
\left\langle T_{\mathrm{FH}}\right\rangle \simeq \sqrt{\frac{\pi N}{2}}
$$

In this limit the first hitting time is dominated by the retracing mechanism.

In Fig. 5 we present analytical results for the mean first hitting time $\left\langle T_{\mathrm{FH}}\right\rangle$ (solid line) of RWs on RRGs as a function of the degree $c$. The analytical results, obtained from Eqs. (29), (36) and (37) are in excellent agreement with the results obtained from computer simulations (circles). In the dilute-network limit $\left\langle T_{\mathrm{FH}}\right\rangle$ quickly increases as $c$ is increased, reaching saturation in the dense-network limit.

\section{The variance of the distribution of first hitting times}

Inserting $r=2$ in Eq. (27) we obtain the second moment $\left\langle T_{\mathrm{FH}}^{2}\right\rangle$ of the distribution of first hitting times, which is given by

$$
\left\langle T_{\mathrm{FH}}^{2}\right\rangle=\sum_{t=0}^{N}(2 t+1) P\left(T_{\mathrm{FH}}>t\right) .
$$

Inserting the tail distribution $P\left(T_{\mathrm{FH}}>t\right)$ from Eq. (24) into Eq. (41), shifting the summation index from $t$ to $t-2$ and replacing the sum by a sum of two integrals, we obtain 
First hitting times of random walks on random reqular graphs

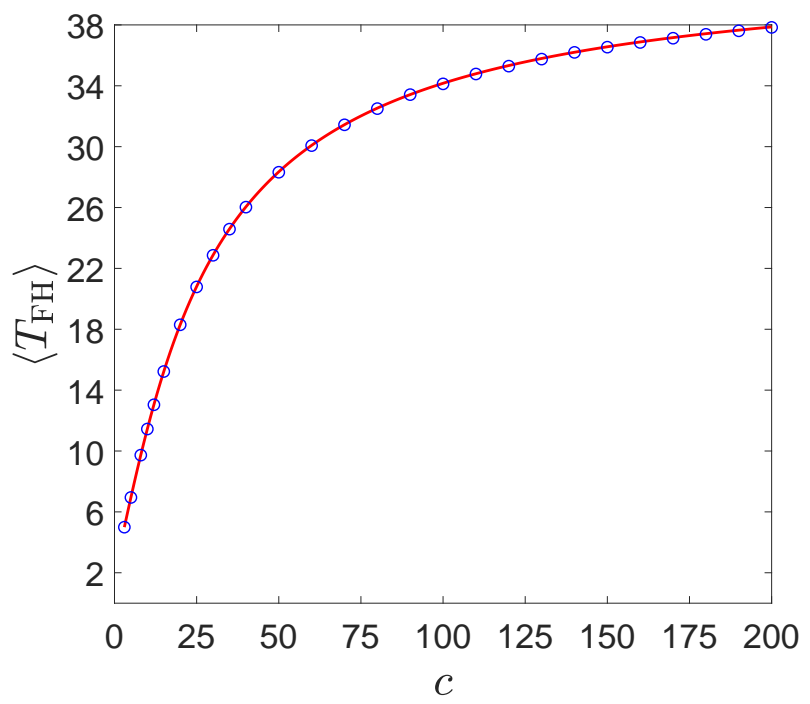

Figure 5. Analytical results for the mean first hitting time $\left\langle T_{\mathrm{FH}}\right\rangle$ of RWs on RRGs of size $N=1000$ (solid line), as a function of the degree $c$. The analytical results, obtained from Eqs. (29), (36) and (37) are in excellent agreement with the results obtained from computer simulations (circles).

$$
\left\langle T_{\mathrm{FH}}^{2}\right\rangle=J_{1}+J_{2}
$$

where

$$
J_{1}=9+7 e^{-\beta}+\int_{3 / 2}^{N-3 / 2}(2 t+5) \exp \left[-\frac{t(t-1)}{2 \alpha^{2}}-\beta t\right] d t
$$

and

$$
J_{2}=-\int_{3 / 2}^{N-3 / 2}(2 t+5) \frac{t(t-1)(2 t-1)}{12\left(\frac{c+2}{c-2}\right) \alpha^{4}} \exp \left[-\frac{t(t-1)}{2 \alpha^{2}}-\beta t\right] d t .
$$

Carrying out the integral in Eq. (43) and taking the large network limit, we obtain

$$
\begin{aligned}
J_{1} & \simeq 9+7 e^{-\beta}+2 \alpha^{2} \exp \left(-\frac{20 \alpha^{2} \beta+15}{8 \alpha^{2}}\right) \\
& -\alpha \sqrt{2 \pi}\left(\alpha^{2} \beta-3\right) e^{\frac{\left(2 \alpha^{2} \beta-1\right)^{2}}{8 \alpha^{2}}}\left[1-\operatorname{erf}\left(\frac{\alpha^{2} \beta+1}{\sqrt{2} \alpha}\right)\right] .
\end{aligned}
$$

Carrying out the integration in Eq. (44) and taking the large network limit, we obtain 


$$
\begin{aligned}
& J_{2} \simeq \frac{1}{12}\left(\frac{c+2}{c-2}\right)\left[4 \alpha^{4} \beta^{3}-4 \alpha^{2} \beta(4 \beta-5)+15 \beta-36\right] e^{-\frac{3}{8 \alpha^{2}}-\frac{3 \beta}{2}} \\
&- \frac{1}{12} \sqrt{\frac{\pi}{2}\left(\frac{c+2}{c-2}\right) \alpha\left[4 \alpha^{4} \beta^{4}-12 \alpha^{2}(\beta-2) \beta^{2}-\beta^{2}-36 \beta+12\right] \times} \\
& e^{\frac{\left(1-2 \alpha^{2} \beta\right)^{2}}{8 \alpha^{2}}}\left[1-\operatorname{erf}\left(\frac{\alpha^{2} \beta+1}{\sqrt{2} \alpha}\right)\right] .
\end{aligned}
$$

In the limit of dilute networks, where $3 \leq c \ll 1.63 \sqrt{N}$, the product $\alpha \beta$ satisfies $\alpha \beta \gg 1$. In this limit the second moment can be approximated by

$$
\begin{aligned}
\left\langle T_{\mathrm{FH}}^{2}\right\rangle & \simeq 9+7 e^{-\beta}-\sqrt{2 \pi} \alpha^{3} \beta e^{\left(\frac{\alpha \beta}{\sqrt{2}}\right)^{2}}\left[1-\operatorname{erf}\left(\frac{\alpha^{2} \beta+1}{\sqrt{2} \alpha}\right)\right] \\
& +2 \alpha^{2} e^{-5 \beta / 2} .
\end{aligned}
$$

In the dense network limit, where $1.63 \sqrt{N} \ll c<N$, the parameter $\beta$ satisfies $\beta \simeq 1 / c$ and $\alpha \beta \ll 1$. As a result, the error function on the right hand side of Eq. (45) becomes negligible and the second moment converges towards

$$
\left\langle T_{\mathrm{FH}}^{2}\right\rangle \simeq 2 N .
$$

The variance of the distribution of first hitting times is given by

$$
\operatorname{Var}\left(T_{\mathrm{FH}}\right)=\left\langle T_{\mathrm{FH}}^{2}\right\rangle-\left\langle T_{\mathrm{FH}}\right\rangle^{2} .
$$

In the dense network limit, the variance converges towards

$$
\operatorname{Var}\left(T_{\mathrm{FH}}\right) \simeq\left(2-\frac{\pi}{2}\right) N .
$$

In Fig. 6] we present analytical results for the variance of the distribution of first hitting times of RWs on RRGs of size $N=1000$ (solid line), as a function of the degree c. The analytical results, obtained from Eq. (49), where $\left\langle T_{\mathrm{FH}}^{2}\right\rangle$ is given by Eqs. (42), (45) and (46) and $\left\langle T_{\mathrm{FH}}\right\rangle$ is given by Eqs. (29), (36) and (37), are in excellent agreement with the results obtained from computer simulations (circles).

\section{Analysis of the backtracking and retracing mechanisms}

The first hitting process consists of two competing scenarios, backtracking and retracing. In each instance of an RW trajectory the first hitting time is determined by the scenario that occurs first. We denote by $P_{\mathrm{BT}}$ the probability that the first hitting event of an RW starting from a random initial node will take place by backtracking. Similarly, we denote by $P_{\text {RET }}$ the probability that the first hitting event will take place by retracing. Since these are the only possible mechanisms of first hitting of RWs, these two probabilities must satisfy $P_{\mathrm{BT}}+P_{\mathrm{RET}}=1$. The conditional probability that the first hitting event 


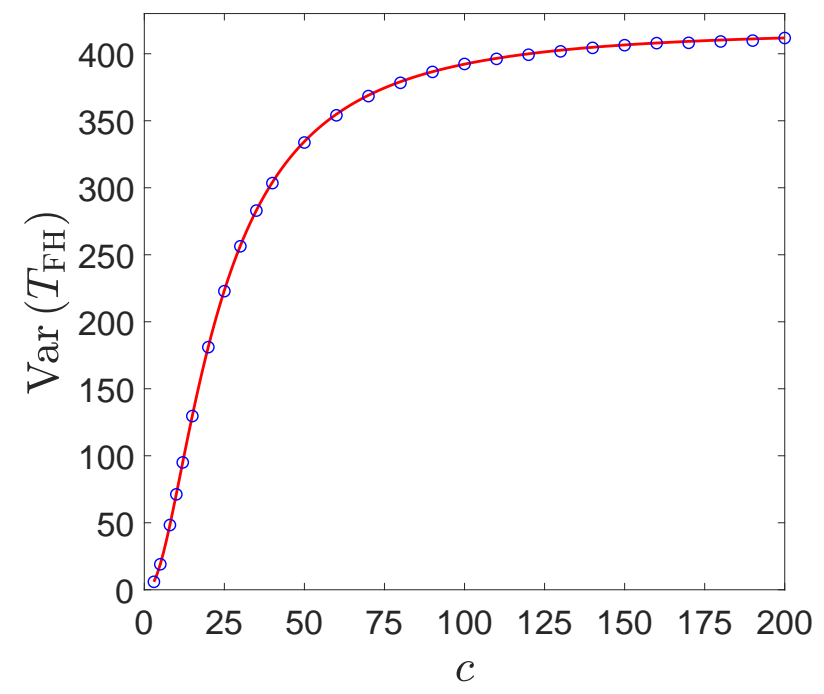

Figure 6. Analytical results for the variance $\operatorname{Var}\left(T_{\mathrm{FH}}\right)$ of the distribution of first hitting times of RWs on RRGs of size $N=1000$ (solid line), as a function of the degree c. The analytical results, obtained from Eq. (49), are in excellent agreement with the results obtained from computer simulations (circles).

will take place at time $t$, given that it occurs via the backtracking scenario, is denoted by $P\left(T_{\mathrm{FH}}=t \mid \mathrm{BT}\right)$. Similarly, the conditional probability that the first hitting event will take place at time $t$ given that it occurs via the retracing scenario is denoted by $P\left(T_{\mathrm{FH}}=t \mid \mathrm{RET}\right)$. The overall distribution of first hitting times can be expressed as a weighted sum of the two conditional distributions, in the form

$$
P\left(T_{\mathrm{FH}}=t\right)=P_{\mathrm{BT}} P\left(T_{\mathrm{FH}}=t \mid \mathrm{BT}\right)+P_{\mathrm{RET}} P\left(T_{\mathrm{FH}}=t \mid \mathrm{RET}\right) .
$$

The first term on the right hand side of Eq. (51) can be written in the form

$$
P_{\mathrm{BT}} P\left(T_{\mathrm{FH}}=t \mid \mathrm{BT}\right)=P\left(T_{\mathrm{FH}}>t-1\right)\left[1-P_{t}(\neg \mathrm{BT})\right]
$$

namely as the probability that the first hitting event will not take place up to time $t-1$ and will occur at time $t$ due to backtracking. Inserting $P_{t}(\neg \mathrm{BT})$ from Eq. (2) into Eq. (52) and summing up over $t$, it is found that the overall probability that the first hitting event will take place via the backtracking scenario is given by

$$
P_{\mathrm{BT}}=\frac{1}{c} \sum_{t=3}^{N+1} P\left(T_{\mathrm{FH}}>t-1\right) .
$$

Comparing with the tail-sum formula (28), the probability $P_{\mathrm{BT}}$ can be expressed in terms of the mean first hitting time, namely

$$
P_{\mathrm{BT}}=\frac{\left\langle T_{\mathrm{FH}}\right\rangle-2}{c} .
$$

Thus, the complementary probability that the first hitting will occur via the retracing mechansim is given by 


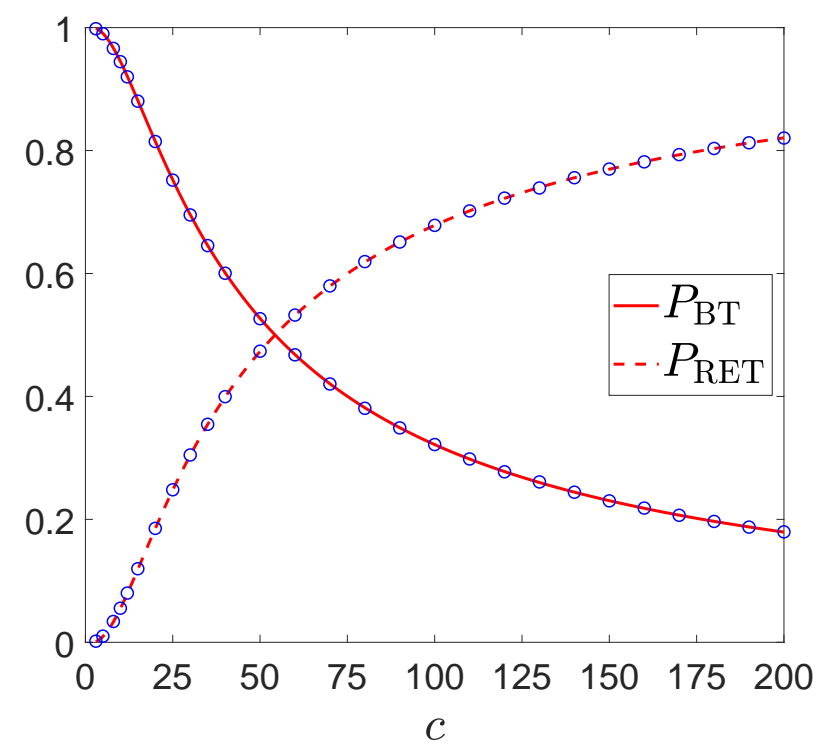

Figure 7. Analytical results for the probabilities $P_{\mathrm{BT}}$ and $P_{\mathrm{RET}}$ that the first hitting event will occur via the backtracking or the retracing scenarios, respectively. The analytical results, obtained from Eqs. (54) and (55), are in excellent agreement with the results obtained from computer simulations (circles).

$$
P_{\mathrm{RET}}=1-\frac{\left\langle T_{\mathrm{FH}}\right\rangle-2}{c} .
$$

Note that Eqs. (54) and (55) are exact. However, inserting $\left\langle T_{\mathrm{FH}}\right\rangle$ from Eq. (29) with $I_{1}$ and $I_{2}$ given by Eqs. (36) and (37), respectively, the results become approximate due to the replacement of the sum in Eq. (28) by integrals.

In Fig. 7 we present the probability $P_{\mathrm{BT}}$ that the first hitting event will occur via the backtracking scenario and the complementary probability $P_{\text {RET }}$ that it will occur via the retracing scenario, as a function of the degree $c$. As expected, the probability $P_{\mathrm{BT}}$ decreases as $c$ is increased while the probability $P_{\text {RET }}$ increases. The crossover from the backtracking-dominated regime of dilute networks to the retracing-dominated regime of dense networks occurs where $P_{\mathrm{BT}}=P_{\mathrm{RET}}=1 / 2$. Using Eqs. (54) and (55) we find that at the crossover point $\left\langle T_{\mathrm{FH}}\right\rangle=(c+4) / 2$. Comparing the right hand side of Eq. (36) to $(c+4) / 2$, it is found that the crossover point occurs at $c \simeq 1.63 \sqrt{N}$.

In Fig. 8 we present a diagram describing the first hitting process of RWs on RRGs. In the regime of dilute networks, where $c \lesssim 1.63 \sqrt{N}$ the first hitting process is dominated by backtracking, while in the regime of dense networks, where $c \gtrsim 1.63 \sqrt{N}$ the first hitting process is dominated by retracing.

The conditional probability $P\left(T_{\mathrm{FH}}=t \mid \mathrm{BT}\right)$, for $t \geq 3$, can be written in the form

$$
P\left(T_{\mathrm{FH}}=t \mid \mathrm{BT}\right)=\frac{P\left(T_{\mathrm{FH}}>t-1\right)\left[1-P_{t}(\neg \mathrm{BT})\right]}{P_{\mathrm{BT}}} .
$$

Inserting $P_{t}(\neg \mathrm{BT})$ from Eq. (2) and $P_{\mathrm{BT}}$ from Eq. (54)), we obtain 


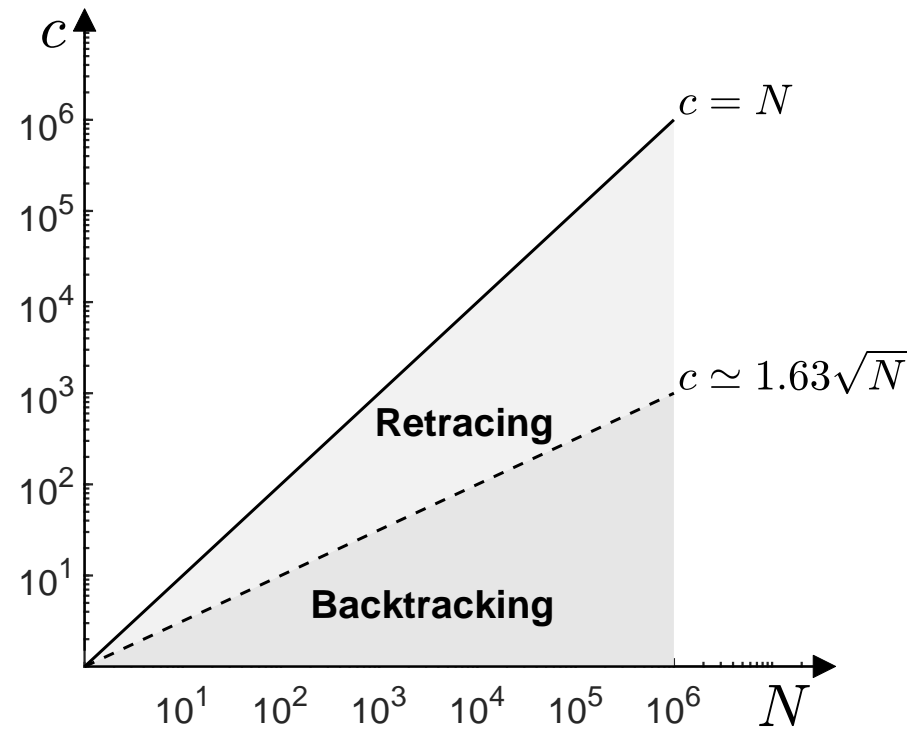

Figure 8. A diagram describing the first hitting process of RWs on RRGs. In the regime of dilute networks, where $c \lesssim 1.63 \sqrt{N}$ the first hitting process is dominated by backtracking, while in the regime of dense networks, where $c \gtrsim 1.63 \sqrt{N}$ the first hitting process is dominated by retracing.

$$
P\left(T_{\mathrm{FH}}=t \mid \mathrm{BT}\right)=\frac{P\left(T_{\mathrm{FH}}>t-1\right)}{\left\langle T_{\mathrm{FH}}\right\rangle-2} .
$$

Focusing on the retracing mechanism, the second term on the right hand side of Eq. (51) can be expressed in the form

$$
P_{\mathrm{RET}} P\left(T_{\mathrm{FH}}=t \mid \mathrm{RET}\right)=P\left(T_{\mathrm{FH}}>t-1\right) P_{t}(\neg \mathrm{BT})\left[1-P_{t}(\neg \mathrm{RET} \mid \neg \mathrm{BT})\right] .
$$

Inserting $P_{t}(\neg \mathrm{BT})$ from Eq. (22), $P_{t}(\neg \mathrm{RET} \mid \neg \mathrm{BT})$ from Eq. (86), and $P_{\text {RET }}$ from Eq. (55) into Eq. (58), we obtain

$$
P\left(T_{\mathrm{FH}}=t \mid \mathrm{RET}\right)=\left(\frac{c-1}{c-\left\langle T_{\mathrm{FH}}\right\rangle+2}\right) \frac{(c-2)(t-3)+1}{(N-2) c-2(t-3)+1} P\left(T_{\mathrm{FH}}>t-1\right) .
$$

In Fig. 9 we present the probabilities $P\left(T_{\mathrm{FH}}=t \mid \mathrm{BT}\right)$ and $P\left(T_{\mathrm{FH}}=t \mid \mathrm{RET}\right)$ that the first hitting process will take place at time $t$, under the condition that it occurs via the backtracking and the retracing scenarios, respectively.

The mean first hitting time under the condition that the first hitting occurs via the backtracking scenario is given by

$$
\mathbb{E}\left[T_{\mathrm{FH}} \mid \mathrm{BT}\right]=\sum_{t=3}^{N+1} t P\left(T_{\mathrm{FH}}=t \mid \mathrm{BT}\right) .
$$

Inserting $P\left(T_{\mathrm{FH}}=t \mid \mathrm{BT}\right)$ from Eq. (57) into Eq. (60), we obtain 

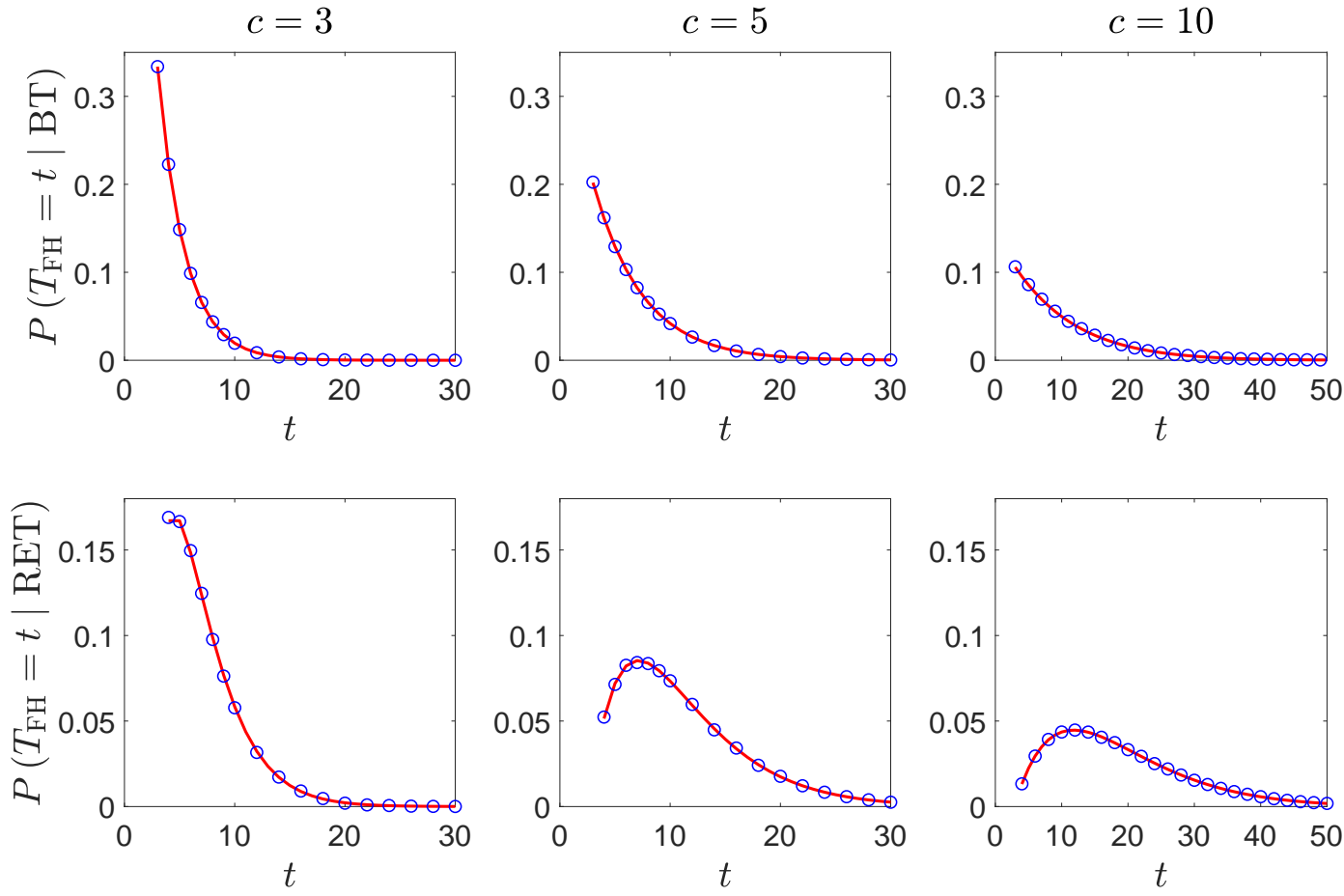

Figure 9. Analytical results for the distribution $P\left(T_{\mathrm{FH}}=t \mid \mathrm{BT}\right)$ and $P\left(T_{\mathrm{FH}}=\right.$ $t \mid$ RET) of first hitting times, conditioned on the first hitting to occur via the backtracking or the retracing scenarios, respectively. The analytical results, obtained from Eqs. (57) and (59), are in excellent agreement with the results obtained from computer simulations (circles).

$$
\mathbb{E}\left[T_{\mathrm{FH}} \mid \mathrm{BT}\right]=\frac{1}{\left\langle T_{\mathrm{FH}}\right\rangle-2} \sum_{t=3}^{N+1} t P\left(T_{\mathrm{FH}}>t-1\right)
$$

Using the tail-sum formula [37], it is found that

$$
\mathbb{E}\left[T_{\mathrm{FH}} \mid \mathrm{BT}\right]=\frac{\left\langle T_{\mathrm{FH}}^{2}\right\rangle+\left\langle T_{\mathrm{FH}}\right\rangle-6}{2\left(\left\langle T_{\mathrm{FH}}\right\rangle-2\right)} .
$$

The mean first hitting time $\left\langle T_{\mathrm{FH}}\right\rangle$ can be expressed as a weighted sum of the form

$$
\left\langle T_{\mathrm{FH}}\right\rangle=\mathbb{E}\left[T_{\mathrm{FH}} \mid \mathrm{BT}\right] P_{\mathrm{BT}}+\mathbb{E}\left[T_{\mathrm{FH}} \mid \mathrm{RET}\right] P_{\mathrm{RET}} .
$$

Inserting $\mathbb{E}\left[T_{\mathrm{FH}} \mid \mathrm{BT}\right]$ from Eq. (62) into Eq. (63) and solving for $\mathbb{E}\left[T_{\mathrm{FH}} \mid \mathrm{RET}\right]$, we obtain

$$
\mathbb{E}\left[T_{\mathrm{FH}} \mid \mathrm{RET}\right]=\frac{\left\langle T_{\mathrm{FH}}^{2}\right\rangle-(2 c-1)\left\langle T_{\mathrm{FH}}\right\rangle-6}{2\left(\left\langle T_{\mathrm{FH}}\right\rangle-c-2\right)} .
$$

In Fig. 10 we present analytical results for the conditional expectation values $\mathbb{E}\left[T_{\mathrm{FH}} \mid \mathrm{BT}\right]$ and $\mathbb{E}\left[T_{\mathrm{FH}} \mid \mathrm{RET}\right]$ of the first hitting time, vs. the degree $c$ given that the first hitting event occurs via the backtracking or the retracing scenario, respectively. The analytical results, obtained from Eqs. (62) and (64), are in excellent agreement with the results obtained from computer simulations (circles). 


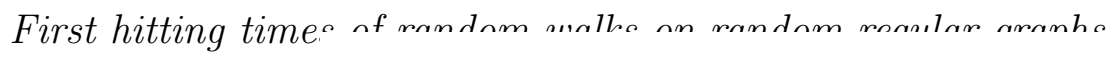

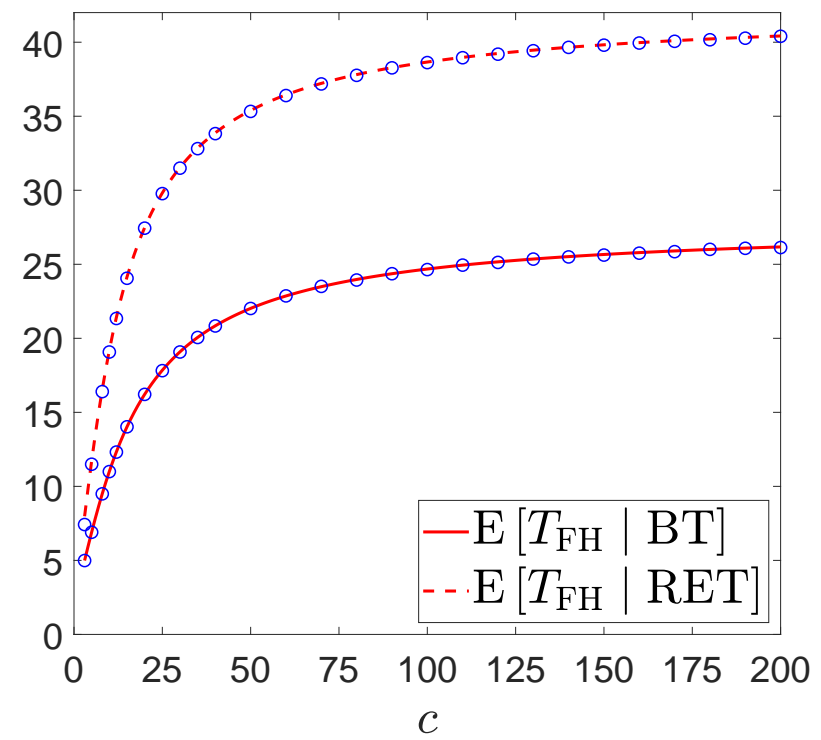

Figure 10. Analytical results for the conditional expectation values $\mathbb{E}\left[T_{\mathrm{FH}} \mid \mathrm{BT}\right]$ and $\mathbb{E}\left[T_{\mathrm{FH}} \mid \mathrm{RET}\right]$ of the first hitting time, vs. the degree $c$ given that the first hitting event occurs via the backtracking or the retracing scenario, respectively. The analytical results, obtained from Eqs. (62) and (64), are in excellent agreement with the results obtained from computer simulations (circles).

\section{Comparing $P\left(T_{\mathrm{FH}}>t\right)$ between RWs on RRGs and RWs on ER networks}

The distribution $P\left(T_{\mathrm{FH}}>t\right)$ of first hitting times of RWs on RRGs is given by Eq. (25)). It can be expressed as a product of a geometric distribution, associated with the backtracking process, and a Rayleigh distribution, associated with the retracing process. The Rayleigh distribution is parameterized by the parameter $\alpha$, while the geometric distribution is parameterized by $\beta$. It is interesting to compare the results obtained above for the distribution $P\left(T_{\mathrm{FH}}>t\right)$ of RWs on RRGs of size $N$ and degree $c$ with the corresponding results for RWs on ER networks of the same size and mean degree $\langle K\rangle=c[26]$. Another interesting comparison is between the distributions of first hitting times of NBWs on RRGs and ER networks. In all these cases, the tail distribution of first hitting times is described by the same functional form as in Eq. (25), but the expressions for $\alpha$ and $\beta$ are different. In Table 1 we present a $2 \times 2$ diagram that provides the parameters $\alpha$ and $\beta$ for RWs on RRGs (upper-left cell), NBWs on RRGs (upper-right cell), RWs on ER networks (lower-left cell) and NBWs on ER networks (lower-right cell).

One difference between RRGs and ER networks is that ER networks include isolated nodes of degree $k=0$. Since an RW starting from an isolated node cannot make even a single move, the initial node of an RW on an ER network is chosen randomly from all the nodes of degree $k \geq 1$. Moreover, isolated nodes are not accessible to the RW at times $t>1$. As a result, for RWs on ER networks the probability of backtracking at any time $t$ is given by $P_{t}(\mathrm{BT})=\left(1-e^{-c}\right) / c$, compared to $1 / c$ in the case of RWs on RRGs [26. This is reflected in the parameter $\beta$ for RWs on ER networks, which 
First hitting times of random walks on random regular graphs

Table 1. The parameters $\alpha$ and $\beta$ of the distribution $P\left(T_{\mathrm{FH}}>t\right)$ [Eq. (25)] for RWs and NBWs on RRGs and on ER networks

\begin{tabular}{|c|c|c|}
\hline & RW & NBW \\
\hline 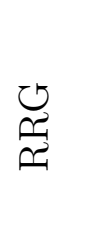 & $\begin{array}{l}\alpha=\sqrt{\left(\frac{c}{c-2}\right) N} \\
\beta=\ln \left(\frac{c}{c-1}\right)\end{array}$ & $\begin{array}{l}\alpha=\sqrt{\left(\frac{c}{c-2}\right) N} \\
\beta=0\end{array}$ \\
\hline 茎 & $\begin{array}{l}\alpha=\sqrt{\left(\frac{c+1}{c}\right) N} \\
\beta=\ln \left(\frac{c}{c-1+e^{-c}}\right)\end{array}$ & $\begin{array}{l}\alpha=\sqrt{\left(\frac{c+1}{c}\right) N} \\
\beta=-\ln \left(1-e^{-c}\right)\end{array}$ \\
\hline
\end{tabular}

includes the $e^{-c}$ term.

The effect of the retracing process on the distribution of first hitting times is described by a Rayleigh distribution parametrized by $\alpha$. In fact, the probability that at any time $t$ the RW will hop into any one of the nodes that were previously visited up to time $t-3$ is given by $1 / \alpha^{2}$. Naively, one expects that the probability to enter a specific node at time $t$ is $1 / N$. However, each visit of a node exhausts two of its edges, which cannot be used to revisit the node in the retracing scenario. Therefore, the probability that at a given time an RW will revisit a specific node that has already been visited two or more time steps earlier is smaller than $1 / N$. In the case of RWs on RRGs it is given by $(1-2 / c) / N$, while in the case of RWs on ER networks it is given by $[1-1 /(c+1)] / N$. These corrections are reflected in the values of $\alpha$ in Table 1 .

In Fig. 11 we compare the analytical results for the distributions $P\left(T_{\mathrm{FH}}>t\right)$ of RWs on RRGs (circles) and ER networks (+) of size $N=1000$ and (a) $c=3$ and (b) $c=5$. For $c=3$, where the first hitting process is dominated by the backtracking scenario, there is a slight difference at short times due to $e^{-c}$ term in the expression for $\beta$ in the ER network. For $c=5$ the results obtained for the two networks are found to be similar. This is due to the fact that as $c$ is increased the $e^{-c}$ term in the expression for $\beta$ of the ER network becomes negligible and it converges towards the expression for $\beta$ of the RRG. This convergence occurs within the dilute network regime, in which the first hitting process is dominated by backtracking.

In order to take a closer look at the retracing process, we consider the distribution of first hitting times in NBWs, in which the backtracking process is suppressed. In the case 
First hitting times of random walks on random reqular araphs
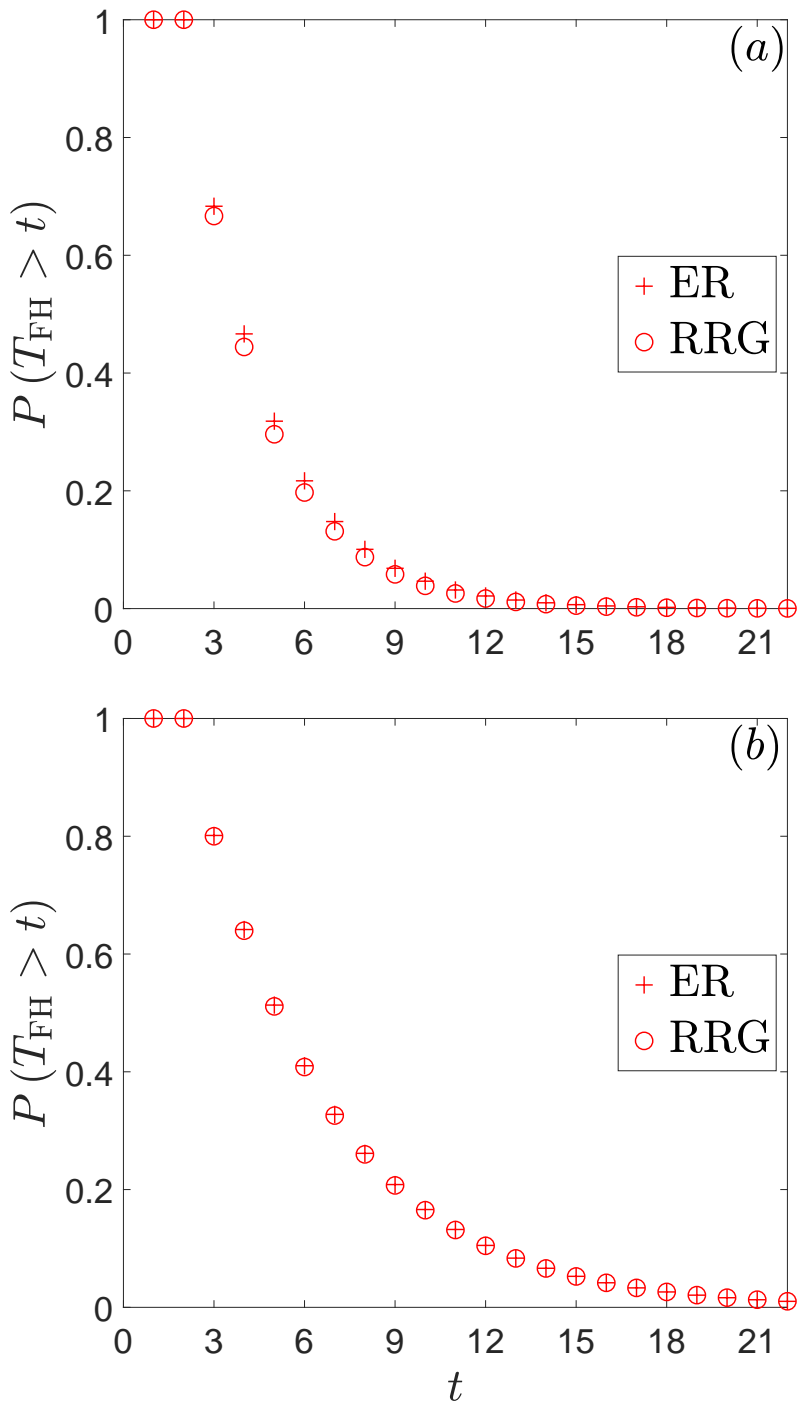

Figure 11. Comparison between the analytical results for the tail distributions $P\left(T_{\mathrm{FH}}>t\right)$ of first hitting times of RWs on RRGs (o) and RWs on ER networks (+). The network size is $N=1000$. The degree is (a) $c=3$ and (b) $c=5$. In both cases, the tail distribution takes the form of Eq. (25), with the parameters $\alpha$ and $\beta$ given in Table 1. Note that the agreement between the analytical results and computer simulations was established in Fig. [3] (for RWs on RRGs) and in Ref. 26] (for RWs on ER networks).

of NBWs on RRGs, upon suppression of the backtracking process, the retracing scenario remains unchanged and the distribution of first hitting times becomes a Rayleigh distribution. In contrast, in the case of NBWs on ER networks the suppression of the backtracking process gives rise to a new mechanism of first hitting, referred to as the trapping scenario. This scenario occurs when the NBW enters a leaf node of degree $k=1$. In the following time step it becomes trapped in the leaf node because the backtracking move into the previous node is not allowed. The distribution of first hitting times of NBWs on ER networks was studied in Ref. [27] and the corresponding expressions for $\alpha$ and $\beta$ are shown in the lower-right cell in Table 1. 

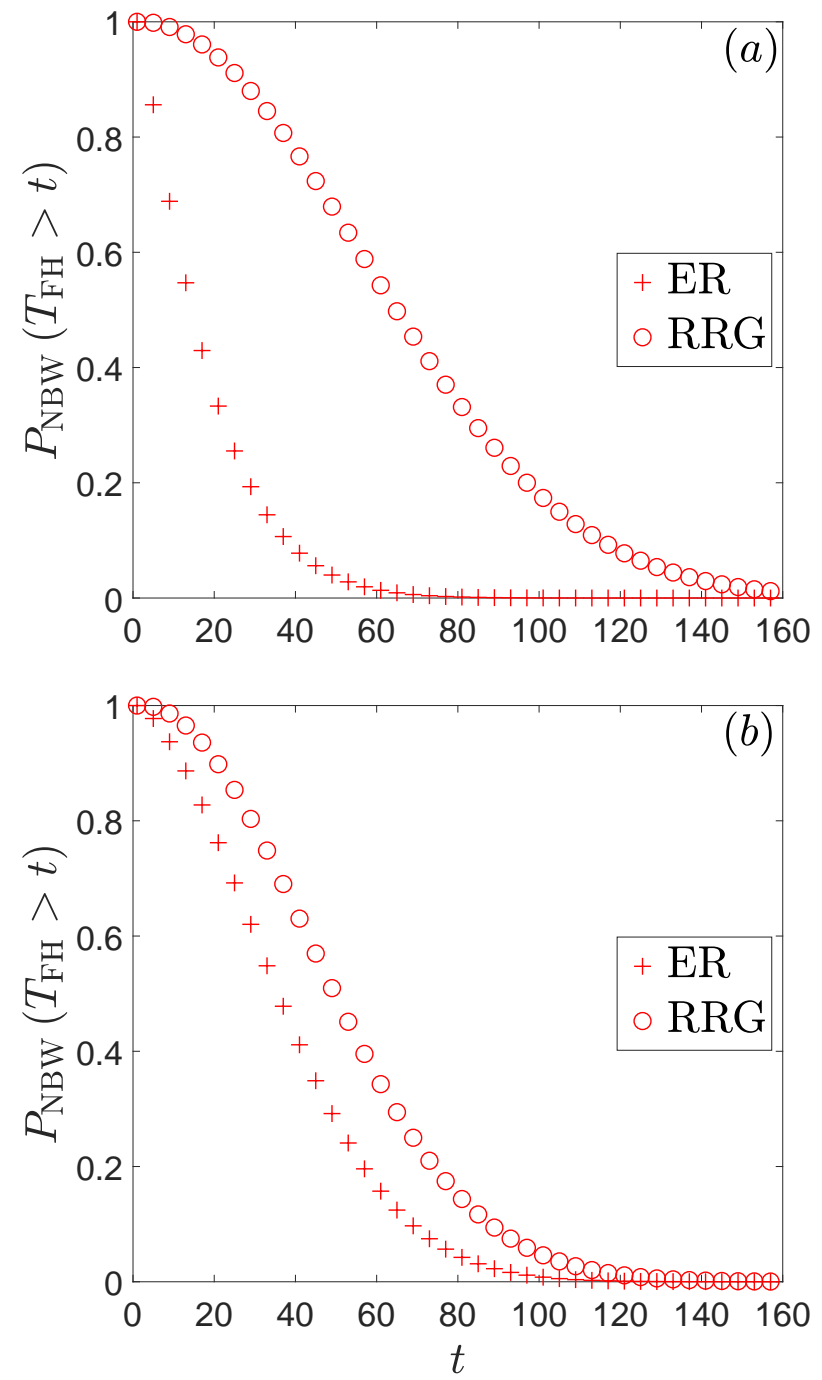

Figure 12. Comparison between the analytical results for the tail distributions $P_{\mathrm{NBW}}\left(T_{\mathrm{FH}}>t\right)$ of first hitting times of NBWs on RRGs (o) and NBWs on ER networks (+). The network size is of size $N=1000$. The degree is (a) $c=3$ and (b) $c=5$. In both cases, the tail distribution takes the form of Eq. (25), with the parameters $\alpha$ and $\beta$ given in Table 1. Note that the agreement between the analytical results and computer simulations was established in Fig. 4 (for NBWs on RRGs) and in Ref. 27] (for NBWs on ER networks).

In Fig. 12 we present a comparison between analytical results for the distributions $P_{\mathrm{NBW}}\left(T_{\mathrm{FH}}>t\right)$ of NBWs on RRGs (circles) and ER networks $(+)$ of size $N=1000$ and (a) $c=3$ and (b) $c=5$. The first hitting times of NBWs on ER networks are found to be much shorter than those obtained for NBWs on RRGs. This contrast is most pronounced for small values of $c$. This is due to the emergence of the trapping scenario which is most effective in the limit of dilute networks. 


\section{Discussion}

Beyond the specific problem of first hitting times of RW on networks, the analysis presented here provides useful insight into the general context of the distribution of life expectancies of humans, animals and machines [39,40]. It illustrates the combination of two lethal hazards, where one hits at a fixed, age-independent rate, while the other increases linearly with age. The first hazard may be considered as an external cause such as an accident while the second hazard involves some aging related degradation which results in an increasing failure rate.

In a more specific context of survival problems, the RW model that terminates upon its first hitting event can be cast in the language of foraging theory as a model describing a wild animal, which is randomly foraging in a random network environment [41]. Each time the animal visits a node it consumes all the food available in this node and needs to move on to one of the adjacent nodes. The model describes rather harsh conditions, in which the regeneration of resources is very slow and the visited nodes do not replenish within the lifetime of the forager. Moreover, the forager does not carry any reserves and in order to survive it must hit a vital node at every time step. More realistic variants of this model have been studied on lattices of different dimensions. It was shown that under slow regeneration rates, the forager is still susceptible to starvation, while above some threshold of the regeneration rate, the probability of starvation diminishes significantly [41]. The case in which the forager carries sufficient resources that enable it to avoid starvation even when it visits up to $S$ non-replenished nodes in a row, was also studied [42,43.

In the dense network limit the first hitting process is dominated by the retracing scenario. As a result, the distribution of first hitting times becomes insensitive to

the degree $c$. In this limit the mean first hitting time scales like $\sqrt{N}$. This can be understood as follows. In this limit, the backtracking probability is very low and thus the backtracking-induced first hitting events become negligible. Instead, retracing becomes the dominant scenario. Due to the very high connectivity, the hopping between adjacent nodes can be considered as the simple combinatorial problem of randomly choosing one node at a time from a set of $N$ nodes, allowing each node to be chosen more than once. In this limit the statistical properties of first hitting times become analogous to those of the birthday problem [44,45]. More specifically, in this limit the probability that $P\left(T_{\mathrm{FH}}>t\right)$ in a network that consists of $N=365$ nodes is equal to the probability that in a party of $t$ participants there will not be even one pair who share the same birthday [44].

\section{Summary}

We presented a statistical analysis of the first hitting times of RWs on RRGs, which may take place either via backtracking or via retracing. The tail distribution $P\left(T_{\mathrm{FH}}>t\right)$ of first hitting times was calculated. It can be expressed as a product of a geometric 
distribution associated with the backtracking process and a Rayleigh distribution which is due to the retracing process. We also obtained closed form expressions for the mean first hitting time $\left\langle T_{\mathrm{FH}}\right\rangle$ and for the variance $\operatorname{Var}\left(T_{\mathrm{FH}}\right)$ of the distribution of first hitting times. The analytical results are found to be in excellent agreement with the results obtained from computer simulations. We obtained analytical results for the probabilities $P_{\mathrm{BT}}$ and $P_{\mathrm{RET}}$ that the first hitting event will occur via the backtracking or retracing scenarios, respectively. We showed that in dilute networks the dominant first hitting scenario is backtracking while in dense networks the dominant scenario is retracing. We also obtained expressions for the conditional distributions of first hitting time, $P\left(T_{\mathrm{FH}}=t \mid \mathrm{BT}\right)$ and $P\left(T_{\mathrm{FH}}=t \mid \mathrm{RET}\right)$, in which the first hitting event occurs via the backtracking or the retracing scenario, respectively. These results provide useful insight into the general problem of survival analysis and the statistics of mortality rates when two or more termination scenarios coexist. We also analyzed the distribution of first hitting times in non-backtracking random walks (NBWs), in which the backtracking process is suppressed and compared the results obtained here for RWs and NBWs on RRGs to earlier results for RWs and NBWs on Erdős-Rényi networks.

This work was supported by the Israel Science Foundation grant no. 1682/18.

\section{References}

[1] Spitzer F 1964 Principles of Random Walk (New York: Springer-Verlag)

[2] Weiss G H 1994 Aspects and Applications of the Random Walk (New York: North Holland)

[3] Berg H C 1993 Random Walks in Biology (Princeton: Princeton University Press)

[4] Ibe O C 2013 Elements of Random Walk and Diffusion Processes (New Jersey: Wiley \& Sons)

[5] Fisher M E 1966 Shape of a self-avoiding walk or polymer chain J. Chem. Phys. 44616

[6] Edwards S F 1965 The statistical mechanics of polymers with excluded volume, Proceedings of the Physical Society $\mathbf{8 5} 613$

[7] De Gennes P G 1979 Scaling Concepts in Polymer Physics (Ithaca: Cornell University Press)

[8] Evans M R and Majumdar S N 2011 Diffusion with stochastic resetting Phys. Rev. Lett. 106 160601

[9] Lopez Millán V M, Cholvi V, Lopez L and Anta A F 2012 A model of self-avoiding random walks for searching complex networks Networks 6071

[10] Lawler G F 2010 Random Walk and the Heat Equation (Providence: American Mathematical Society)

[11] Lawler G F and Limic V 2010 Random Walk: A Modern Introduction (Cambridge: Cambridge University Press)

[12] ben-Avraham D and Havlin S 2000 Diffusion and Reactions in Fractals and Disordered Systems (Cambridge: Cambridge University Press)

[13] Noh D J and Rieger H 2004 Random walks on complex networks Phys. Rev. Lett. 92118701

[14] Havlin S and Cohen R 2010 Complex Networks: Structure, Robustness and Function (Cambridge University Press, New York)

[15] Newman M E J 2018 Networks: an Introduction, $2^{\text {nd }}$ Ed. (Oxford: Oxford University Press)

[16] Pastor-Satorras R and Vespignani A 2001 Epidemic spreading in scale-free networks Phys. Rev. Lett. 863200

[17] Barrat A, Barthélemy M and Vespignani A 2012 Dynamical Processes on Complex Networks (Boston: Cambridge University Press) 
[18] De Bacco C, Majumdar S N and Sollich P 2015 The average number of distinct sites visited by a random walker on random graphs J. Phys. A 48205004

[19] Montroll E W and Weiss G H 1965 Random Walks on Lattices II J. Math. Phys. 6167

[20] Herrero C P and Saboyá M 2003 Self-avoiding walks and connective constants in small-world networks Phys. Rev. E 68026106

[21] Herrero C P 2005 Kinetic growth walks on complex networks J. Phys. A 384349

[22] Redner S 2001 A Guide to First Passage Processes (Cambridge: Cambridge University Press)

[23] Note that occasionally in the literature the term "first hitting time" is used as an alternative to the term "first passage time".

[24] Kahn J D, Linial N, Nisan N and Saks M E 1989 On the cover time of random walks on graphs J. Theor. Probab. 2121

[25] Alon N, Benjamini I, Lubetzky E and Sodin S 2007 Non-backtracking random walks mix faster Commun. Contemp. Math. 9585

[26] Tishby I, Biham O and Katzav E 2017 The distribution of first hitting times of random walks on Erdős-Rényi networks J. Phys. A 50115001

[27] Tishby I, Biham O and Katzav E 2017 The distribution of first hitting times of nonbacktracking random walks on Erdős-Rényi networks J. Phys. A 50205003

[28] Molloy M and Reed A 1995 A critical point for random graphs with a given degree sequence Rand. Struct. Alg. 6161

[29] Molloy M and Reed A 1998 The Size of the Giant Component of a Random Graph with a Given Degree Sequence Combinatorics, Probability and Computing 7295

[30] Newman M E J, Strogatz S H and Watts D J 2001 Random graphs with arbitrary degree distributions and their applications, Phys. Rev. E 64026118

[31] Erdős P and Rényi A 1959 On random graphs. I Publicationes Mathematicae (Debrecen) 6290

[32] Erdős P and Rényi A 1960 On the evolution of random graphs Publ. Math. Inst. Hung. Acad. Sci. 517

[33] Erdős P and Rényi A 1961 On the evolution of random graphs. II Bull. Inst. Int. Stat. 38343

[34] Bonneau H, Hassid A, Biham O, Kühn R and Katzav E 2017 Distribution of shortest cycle lengths in random networks Phys. Rev. E 96062307

[35] Pathria R K and Beale P D 2011 Statistical Mechanics 3rd Edition (Amsterdam: Academic Press)

[36] Papoulis A, Pillai S and Unnikrishna S 2002 Probability, Random Variables and Stochastic Processes (Boston: McGraw-Hill)

[37] Pitman J 1993 Probability (New York: Springer-Verlag)

[38] Olver F W J, Lozier D M, Boisvert R R and Clark C W 2010 NIST Handbook of Mathematical Functions (Cambridge: Cambridge University Press)

[39] Finkelstein M 2008 Failure Rate Modeling for Reliability and Risk (London: Springer-Verlag)

[40] Gavrilov L A and Gavrilova N S 2001 The reliability theory of aging and longevity J. theor. Biol 213527

[41] Chupeau M, Bénichou O and Redner S 2016 Universality classes of foraging with resource renewal Phys. Rev. E 93032403

[42] Bénichou O and Redner S 2014 Depletion-Controlled Starvation of a Diffusing Forager Phys. Rev. Lett 113238101

[43] Bénichou O, Chupeau M and Redner S 2016 Role of depletion on the dynamics of a diffusing forager J. Phys. A 49394003

[44] Knight W and Bloom D M 1973 A birthday problem Amer. Math. Monthly 801141

[45] Flajolet P, Gardy D and Thimonier L 1992 Birthday paradox, coupon collectors, caching algorithms and self-organizing search Discrete Applied Mathematics 39207 